\title{
Response of a Shape Memory Alloy Beam Model under Narrow Band Noise Excitation
}

\author{
Gen Ge \\ School of Mechanical Engineering, Tianjin Polytechnic University, Tianjin 300072, China \\ Correspondence should be addressed to Gen Ge; gegenroot@126.com
}

Received 29 September 2013; Revised 22 January 2014; Accepted 5 February 2014; Published 9 March 2014

Academic Editor: Shabnam Arbab Chirani

Copyright (C) 2014 Gen Ge. This is an open access article distributed under the Creative Commons Attribution License, which permits unrestricted use, distribution, and reproduction in any medium, provided the original work is properly cited.

To describe the hysteretic nonlinear characteristic of the strain-stress relation of shape memory alloy (SMA), a Van-der-Pol hysteretic cycle is applied to simulate the hysteretic loops. Then, the model of a simply supported SMA beam subject to transverse narrow band noise excitation with nonlinear damping was proposed. The deterministic and the stochastic responses are studied, respectively, applying the multiple scale method. The stability of the steady state responses is analyzed by Floquet theory and the moment method. The numerical simulation results quite agree with the theoretical analysis.

\section{Introduction}

Shape memory alloy (SMA) is a kind of smart materials and is applied in the engineering field widely. There are various mechanical models of SMA proposed in recent decades [13]. Most of them are based on thermodynamics theory and micromechanics theory. However, those mechanical models of SMA are mostly shown as equations with subsection function which are hard to be analyzed. Furthermore, SMA beams are widely used in mechanical engineering as sensors and actors. It is of great importance to study the vibrating character of the SMA beam. In the recent decades, some impressive works have been done on the nonlinear vibrating of the SMA beam [4-9]. Many interesting phenomena such as bifurcation, period doubling, jumping, and chaos can be observed. Anyway, the studies mentioned above are all about deterministic excitations. Stochastic excitations widely exist in engineering applications, such as wind loads and seismic waves. By now, few has been done to explore the dynamical responses induced by stochastic excitation. For instance, Yan and Nie [10] studied a SMA superelastic subjected to stationary random excitations.

In this paper, we focus on a SMA beam under narrow band noise excitation. Narrow band noise is a harmonic function with constant amplitude and random frequency, which is a reasonable model for the random excitation or response to engineering systems compared with the harmonic excitations. In Section 2, a Van-der-Pol hysteretic cycle is applied to simulate the hysteretic character of SMA based on the experimental data, and the Galerkin method is used to obtain the one-degree-freedom ordinary differential equation governing the motion of a simply supported SMA beam. Then the multiple scale method is applied to simplify the original function and get the stationary state response in Section 3. Furthermore, in Section 4, the Ito-type stochastic differential equation is obtained by linearization, and the responses and stability of both the noise-free system and the noise included system are studied using Floquet theory and the moment method. Finally, the numerical simulation is carried out to prove the theoretical analysis.

\section{Modeling of the SMA Beam}

The experimental hysteretic nonlinearity strain-stress curve of SMA [11] is shown in Figure 1. The length of the Ti-Ni SMA specimens is $14 \mathrm{~cm}$, the diameter of the specimens is $1.94 \mathrm{~mm}$, and transformation temperature is $-10^{\circ} \mathrm{C}$. The experimental equipment is Instron 8501 plus type fatigue testing machine. The testing temperature is set at $25^{\circ} \mathrm{C}$, the exciting frequency is set as $1 \mathrm{~Hz}$, and the cycle number is set as 40 . According to [11], it is clear that when the cycle number is limited, the shape of the hysteretic loop does not change much, even the exciting 


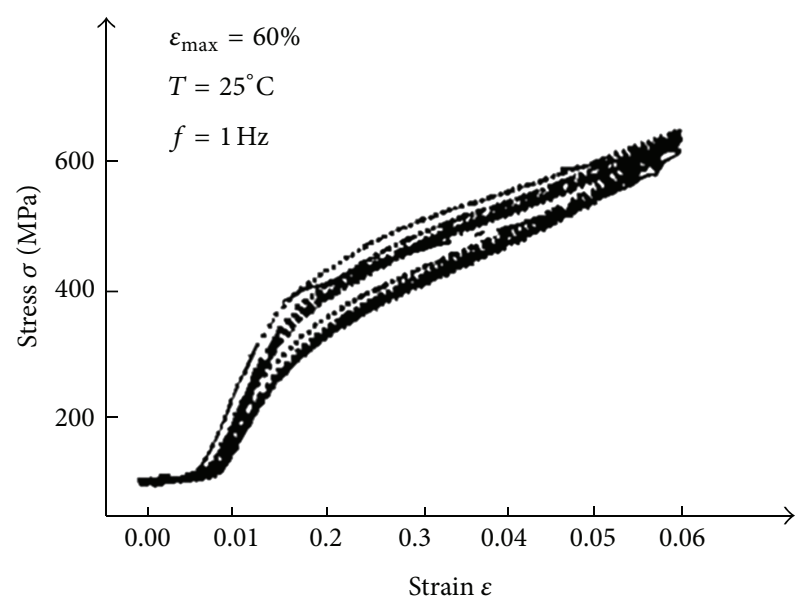

FIGURE 1: The experimental stain-stress curve.

frequency is different. As we know, the SMA is usually used as damper in some structure, for example, aseismic structure. In fact, the effective imposing time of aseismic structure is less than 20 seconds, so the number of the hysteretic loops is limited. Then we can assume that the shape of the hysteretic loop remains unchanged.

Now, Van-der-Pol hysteretic cycle model was introduced to describe the hysteretic characteristic of SMA. Before the numerical simulation, some assumptions must be given. Firstly, because the difference of the curves is small when the cycle number is limited, we take the average stress value as the final experimental data (see in Figure 2). Secondly, the residual strain is obviously small, so it could be ignored for convenience. Thirdly, considering that the bending deformation is small in most working conditions, the tensilecompression asymmetry of SMA behavior is also ignored. In short, in this paper we focus on the method of simulating the hysteretic loop. The initial Van-der-Pol hysteretic cycle model describes hysteretic cycle which is symmetrical about the initial point $(0,0)$. Since the curve is obtained by isostrain rate tensile test, we define a parameter $\dot{x}$ as loading rate, which is chosen as 1 while loading ( -1 as unloading). This parameter just has mathematical meaning. Then the model can be shown as follows:

$$
\begin{aligned}
y= & f(x)=f_{0}(x)+a\left[1-\left(\frac{x}{b}\right)^{2}\right] \dot{x}=b_{1} x \\
& +b_{2} x^{3}+a\left[1-\left(\frac{x}{b}\right)^{2}\right] \dot{x},
\end{aligned}
$$

where $f_{0}(x)$ is skeleton curve of hysteretic cycle and $a$ and $b$ are coefficients. The parameters $a$ and $b$ are used to change the shape of the hysteretic loop. Supposing the strain-stress curve of SMA is symmetrical about the point $G\left(\varepsilon_{0}, \sigma_{0}\right)$, one may substitute $y=\sigma-\sigma_{0}$ and $x=\varepsilon-\varepsilon_{0}$ into (1). Considering the residual strain of SMA should be avoided in simulation,that is, when $\varepsilon=0$, there is $\sigma=0$. Then the final expression of the stress-stain relation of SMA is

$$
\sigma=a_{1} \varepsilon+a_{2} \varepsilon^{2}+a_{3} \varepsilon^{3}+\left(a_{4} \varepsilon-a_{5} \varepsilon^{2}\right) \dot{\varepsilon}
$$

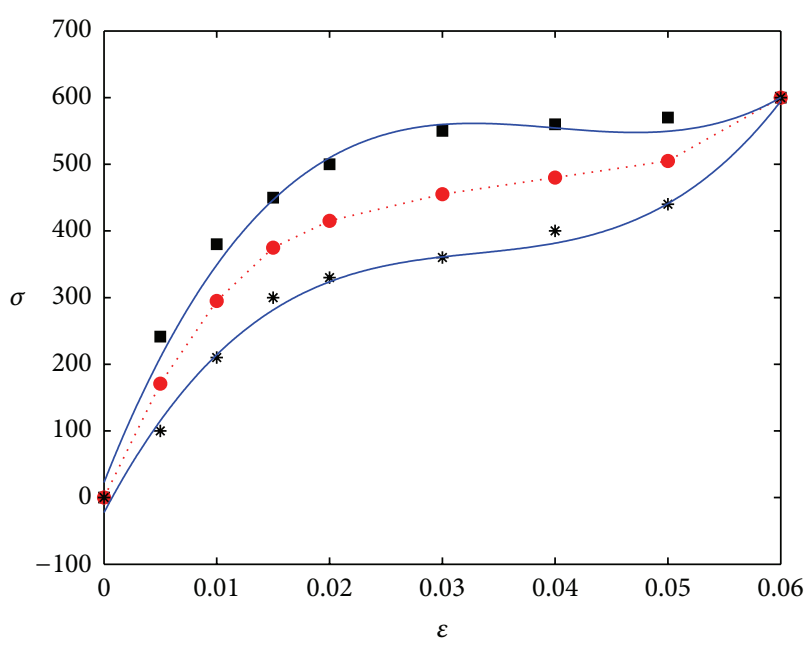

- Experimental loading data $\quad * \quad$ Unloading data

-. Skeleton curve _ Fitting curve

FIGURE 2: The fitting diagram of stain-stress curve.

where $a_{1}=b_{1}+3 b_{2} \varepsilon_{0}^{2}, a_{2}=-3 b_{2} \varepsilon_{0}, a_{3}=b_{2}, a_{4}=2 a / \varepsilon_{0}$, and $a_{5}=a / \varepsilon_{0}^{2}$.

Then the strain-stress curve of SMA obtained by numerical fitting can be shown in Figure 2.

One hinged-hinged SMA beam is shown in Figure 3 with thickness $h$, length $L$, and width $b$. The axial force may be expressed in the form $N=p_{0}$. The transverse narrow band noise excitation is in the form $F \eta(t) \sin ((\pi / L) x)$. Here, only the first vibration mode is considered, as we think it will have enough accuracy for most engineering applications. $\eta(t)$ is in the form of $\cos \left(\Omega_{0} t+\delta^{*} W(t)\right)$, where $\Omega_{0}$ denotes the center frequency, $W(t)$ denotes the standard Wiener process, and $\delta^{*}$ denotes the small strength of the $W(t)$.

Considering the boundary conditions at $x=0, w=0$, $w_{x x}=0$ and at $x=L, w=0, w_{x x}=0$, the transverse deflection $w$ is expressed as $w(t, x)=u(t) \sin (\pi x / L)$, where $u(t)$ is the amplitude. Consider the small geometrical deformation condition $\varepsilon=-y \partial^{2} w / \partial x^{2}$.

The bending moment $M$ is presented as

$$
\begin{aligned}
M= & b \int_{-h / 2}^{h / 2}-y\left[a_{1} \varepsilon+a_{2} \varepsilon^{2}+a_{3} \varepsilon^{3}+\left(a_{4} \varepsilon-a_{5} \varepsilon^{2}\right) \dot{\varepsilon}\right] d y \\
= & -I_{1} a_{1} \pi^{2} u \sin \left(\frac{\pi}{L} x\right)-I_{3} a_{3} \pi^{6} u^{3} \sin ^{3}\left(\frac{\pi}{L} x\right) \\
& +I_{3} a_{5} \pi^{6} u^{2} \sin ^{3}\left(\frac{\pi}{L} x\right) \dot{u},
\end{aligned}
$$

where $I_{1}=b h^{3} /\left(12 L^{2}\right)$ and $I_{3}=b h^{5} / 80 L^{6}$.

The dynamical motion equation [8] is expressed as

$$
\frac{\partial^{2} M}{\partial x^{2}}+\frac{N \partial^{2} w}{\partial x^{2}}+\frac{c \partial w}{\partial t}+\frac{\rho A \partial^{2} w}{\partial t^{2}}=F \eta(t),
$$

where $c$ is the linear damping coefficient, $\rho$ is the density of the SMA, and $A$ is the area of the cross-section of the beam. Substituting (2) and (3) into (4) and applying the Galerkin 


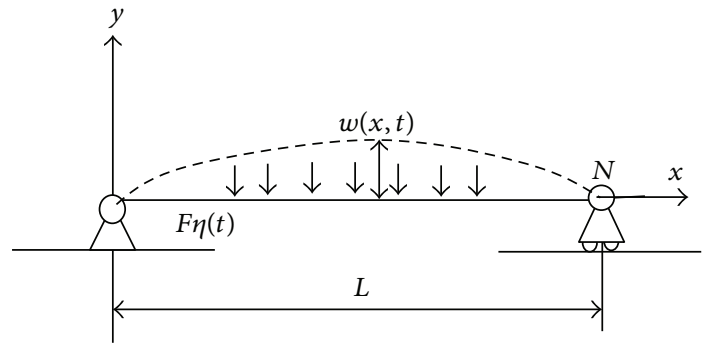

FIgURE 3: The hinged-hinged SMA beam.

approach, one obtains a differential equation of motion governing the deflection of a beam as follows:

$$
\begin{aligned}
\ddot{u}+ & \frac{a_{1} I_{1} \pi^{4}-p_{0} \pi^{2}}{\rho A L^{2}} u+\left(\frac{c}{\rho A}-\frac{3 a_{5} I_{3} \pi^{8}}{4 \rho A L^{2}} u^{2}\right) \dot{u} \\
& +\frac{3 a_{3} I_{3} \pi^{8}}{4 \rho A L^{2}} u^{3}=\frac{4}{\pi \rho A} F \cos \left(\Omega_{0} t+\delta^{*} W(t)\right) .
\end{aligned}
$$

Considering $\omega_{0}=\sqrt{\left(\left(a_{1} I_{1} \pi^{4}-p_{0} \pi^{2}\right) / \rho A l^{2}\right)}, t^{*}=\omega_{0} t, x=$ $u / L, \Omega=\Omega_{0} / \omega_{0}$, and $\bar{\delta}=\sqrt{\omega_{0}} \delta^{*}$, it is easy to know that

$$
\dot{x}\left(t^{*}\right)=\frac{d x}{d t^{*}}=\frac{1}{\omega_{0} L} \frac{d u}{d t}, \quad \ddot{x}\left(t^{*}\right)=\frac{1}{\omega_{0}^{2} L} \frac{d u}{d t} .
$$

Substituting (6) and no dimensional variables into (5) and dropping the over star yield the dimensionless function as follows:

$$
\ddot{x}+x+\varepsilon\left(2 \mu-\beta x^{2}\right) \dot{x}+\varepsilon \alpha x^{3}=\varepsilon f \cos (\Omega t+\bar{\delta} W(t)),
$$

where $\mu=\left(c / 2 \rho A \omega_{0}\right), \beta=\left(3 a_{5} I_{3} \pi^{8} / 4 \rho A \omega_{0}\right), \alpha=\left(3 a_{3} I_{3} \pi^{8} /\right.$ $\left.4 \rho A \omega_{0}^{2}\right), f=\left(4 / \pi \rho A \omega_{0}^{2} L\right) F$, and $\varepsilon$ is the mark for small perturbation terms.

\section{Multiple Scale Method}

A uniformly approximate solution of (2) is sought in the form

$$
x(t, \varepsilon)=x_{0}\left(T_{0}, T_{1}\right)+\varepsilon x_{1}\left(T_{0}, T_{1}\right)+\cdots,
$$

where $T_{0}=t, T_{1}=\varepsilon t$ are fast and slow scale, respectively.

In this paper only the first-order uniform expansion of the solution $x_{0}\left(T_{0}, T_{1}\right)$ is discussed. By denoting $D_{0}=\partial / \partial T_{0}$ and $D_{1}=\partial / \partial T_{1}$, the ordinary-time derivatives can be transformed into partial derivatives as

$$
\frac{d}{d t}=D_{0}+\varepsilon D_{1}+\cdots \frac{d^{2}}{d t^{2}}=D_{0}^{2}+\varepsilon 2 D_{0} D_{1}+\cdots .
$$

Substituting (8) and (9) into (2) and comparing coefficients of $\varepsilon$ with equal powers, one obtains the following equations:

$$
\begin{aligned}
D_{0}^{2} x_{0}+x_{0}= & 0, \\
D_{0}^{2} x_{1}+x_{1}= & -2 D_{0} D_{1} x_{0}-\alpha x_{0}^{3} \\
& -\left(2 \mu-\beta x_{0}^{2}\right) D_{0} x_{0}+f \cos \left(\Omega T_{0}+\bar{\delta} W(t)\right) .
\end{aligned}
$$

The general solution of (10) can be expressed as

$$
x_{0}\left(T_{0}, T_{1}\right)=A\left(T_{1}\right) \exp \left(i T_{0}\right)+\mathrm{cc},
$$

where the cc is the complex conjugate of its preceding terms and $A\left(T_{1}\right)$ is the slowly varying amplitude of the response. Substituting (9) and (11) into (10), one obtains

$$
\begin{aligned}
D_{0}^{2} x_{1}+x_{1}= & -2 D_{1} A i \mathrm{e}^{i T_{0}} \\
& -\alpha\left(A^{3} \mathrm{e}^{i 3 T_{0}}+3 A^{2} \bar{A} \mathrm{e}^{i T_{0}}\right)-2 \mu i A \mathrm{e}^{i T_{0}} \\
& +\beta\left(i A^{3} \mathrm{e}^{i 3 T_{0}}+i A^{2} \bar{A} \mathrm{e}^{i T_{0}}\right) \\
& +\frac{1}{2} f \mathrm{e}^{i\left(T_{0}+\delta W\left(T_{1}\right)\right)}+\mathrm{cc}
\end{aligned}
$$

where the over bar stands for the complex conjugate, $\delta=$ $\bar{\delta} / \sqrt{\varepsilon}$. For the Wiener process $W(t), E[W(t)]=0, E\left[W^{2}(t)\right]=$ $t$, where $E[\cdot]$ denotes the expectation, one has

$$
\bar{\delta} W(t)=\frac{\bar{\delta}}{\sqrt{\varepsilon}} W(\varepsilon t)=\delta W\left(T_{1}\right) .
$$

In this paper we focus on the primary resonances of the system (2). Introducing the detuning parameter $\sigma_{0}$ as $\Omega=$ $1+\varepsilon \sigma_{0}$, one has

$$
\Omega t=T_{0}+\sigma_{0} T_{1}
$$

Using (14) and eliminating the secular terms of (12) yield

$$
-2 D_{1} A i-3 \alpha A^{2} \bar{A}-2 \mu i A+i \beta A^{2} \bar{A}+\frac{1}{2} f \mathrm{e}^{i\left(\sigma_{0} T_{1}+\delta W\left(T_{1}\right)\right)}=0 .
$$

Expressing $A\left(T_{1}\right)$ into the polar form

$$
A\left(T_{1}\right)=a\left(T_{1}\right) \exp \left[i \varphi\left(T_{1}\right)\right] .
$$

Substituting (16) into (15) and separating the real and imaginary parts of (15), one obtains

$$
\begin{aligned}
& \frac{d a}{d T_{1}}=-\mu a+\frac{1}{8} \beta a^{3}-\frac{1}{2} f \sin \eta, \\
& a \frac{d \eta}{d T_{1}}=-\sigma_{0} a+\frac{3}{8} \alpha a^{3}-\frac{1}{2} f \cos \eta-\delta a W^{\prime}\left(T_{1}\right),
\end{aligned}
$$

where $\eta=\varphi-\sigma_{0} T_{1}-\delta W^{\prime}\left(T_{1}\right)$ and (17) is the first-order equation governing the modulation of the amplitude and phase.

The steady state response $\left(d a / d T_{1}\right)=\left(d \eta / d T_{1}\right)=0$ is decided by

$$
\begin{aligned}
& -\mu \bar{a}+\frac{1}{8} \beta \bar{a}^{3}=\frac{1}{2} f \sin \bar{\eta}, \\
& -\sigma_{0} \bar{a}+\frac{3}{8} \alpha \bar{a}^{3}-\delta \bar{a} W^{\prime}(T)=\frac{1}{2} f \cos \bar{\eta}
\end{aligned}
$$

where $\bar{a}$ and $\bar{\eta}$ denote the steady amplitude and phase. 


\section{Responses and Stability}

Then the Floquet theory is applied to investigate the stability of the steady state response by linearization. Assuming $a=$ $\bar{a}+\Delta a \eta=\bar{\eta}+\Delta \eta$, where $\Delta a, \Delta \eta$ are the small perturbation terms, substituting the above equations into (17), and ignoring the high order terms, the linearization equation (20) is expressed as

$$
\begin{aligned}
\frac{d \Delta a}{d T_{1}}= & -\mu \Delta a+\frac{3}{8} \beta \bar{a}^{2} \Delta a-\frac{1}{2} f \cos \bar{\eta} \Delta \eta, \\
\frac{d \Delta \eta}{d T_{1}}= & \left(\frac{3}{4} \alpha \bar{a}+\frac{1}{\bar{a}^{2}} \frac{1}{2} f \cos \bar{\eta}-\delta W^{\prime}(t)\right) \Delta a \\
& +\frac{1}{\bar{a}} \frac{1}{2} f \sin \bar{\eta} \Delta \eta .
\end{aligned}
$$

Substituting (18) into (19) yields the Ito-type stochastic equation:

$$
\begin{aligned}
& d \Delta a=\left[\left(-\mu+\frac{3}{8} \beta \bar{a}^{2}\right) \Delta a+\left(\sigma_{0} \bar{a}-\frac{3}{8} \alpha \bar{a}^{3}\right) \Delta \eta\right] d T_{1}, \\
& d \Delta \eta=\left[\left(\frac{9 \alpha}{8} \bar{a}-\frac{\sigma}{\bar{a}}\right) \Delta a+\left(-\mu+\frac{1}{8} \beta \bar{a}^{2}\right) \Delta \eta\right] d T_{1}-\delta d W(t),
\end{aligned}
$$

where $d W(t)$ denotes the standard Gauss white noise.

4.1. Noise-Free Condition. Assuming $\delta$ is sufficiently small, we determine the response of system (2) for the noise-free condition $(\delta=0)$. In this case, (18) can lead to the following frequency response formula:

$$
G=\left(-\mu \bar{a}+\frac{1}{8} \beta \bar{a}^{3}\right)^{2}+\left(-\sigma_{0} \bar{a}+\frac{3}{8} \alpha \bar{a}^{3}\right)^{2}-\frac{f^{2}}{4}=0 .
$$

Obviously, in some proper parametrical conditions, there are multiple solutions coexisting of (21). Some of them are stable and the others are unstable. It is necessary to know how the system's parameters influence the numbers of the solution. The singularity theory [12] is applied to study the condition for multisolutions of (21). Choosing (21) as the bifurcation function and detuning parameter $\sigma_{0}$ amplitude $f$ as universal unfolding parameters, the Hysteresis point set is given by

$$
G\left(\bar{a}, \sigma_{0}, f\right)=G_{\bar{a}}\left(\bar{a}, \sigma_{0}, f\right)=G_{\bar{a} a}\left(\bar{a}, \sigma_{0}, f\right)=0
$$

which yields

$$
\frac{3}{16} f^{2}\left(\mu \beta+3 \alpha \sigma_{0}\right)-\left(\mu^{2}+\sigma_{0}^{2}\right)^{2}=0
$$

When the parameter $\sigma_{0}$ is increased across the line expressed by (23) for a set of given other parameters, the number of the solutions of (21) bifurcates from one to three.

For the triple-solution condition, it is necessary to know the stable conditions for the steady state solutions. The Jacoby matrix is a usually used way to judge the stability, which is expressed as follows:

$$
\operatorname{det}\left|\begin{array}{cc}
\left.-\mu+\frac{3}{8} \beta \bar{a}^{2}\right)-\lambda & \sigma_{0} \bar{a}-\frac{3}{8} \alpha \bar{a}^{3} \\
\frac{9 \alpha \bar{a}}{8}-\frac{\sigma_{0}}{\bar{a}} & \left(\frac{\beta}{8} \bar{a}^{2}-\mu\right)-\lambda
\end{array}\right|=0 .
$$

The characteristic equation can be expressed as

$$
\lambda^{2}-P \lambda+Q=0
$$

where

$$
\begin{aligned}
& P=\frac{1}{2} \beta \bar{a}^{2}-2 \mu \\
& Q=\left(\mu^{2}+\sigma_{0}^{2}\right)-\left(\frac{\mu \beta+3 \alpha \sigma_{0}}{2}\right) \bar{a}^{2}+\left(\frac{27 \alpha^{2}+3 \beta^{2}}{64}\right) \bar{a}^{4} .
\end{aligned}
$$

The roots of (25) are

$$
\lambda_{1,2}=\frac{P \pm \sqrt{P^{2}-4 Q}}{2} .
$$

Obviously, the stable condition of the system (19) without noise is $P<0, Q>0$ (condition 1), which means that the solution is realizable by numerical simulation. And the condition for existing complex roots is $P^{2}-4 Q<0$ (condition 2 ), which means that there exist periodical vibrations.

In this paper, the parameters are chosen as $\mu=0.1$, $\beta=0.1, \alpha=0.5, f=0.2$, and $\varepsilon=0.1$. By function (23), one knows that if the detuning parameter $\sigma_{0}$ is larger than 0.193 , the number of steady state solutions bifurcates from one to three. In Figure 4(a), one can observe clearly that when $\sigma_{0}$ is chosen as 0.22 , there are three steady state solutions coexisting. The largest one denoted as $\bar{a}_{L}$ and the smallest one denoted as $\bar{a}_{S}$ are stable sinks, while the middle one denoted as $\bar{a}_{u}$ is unstable saddle. In Figure $4(\mathrm{~b})$, when $\sigma_{0}$ is chosen as 0.18 , there is only steady state solution which is a stable sink in the phase plane.

The numerical simulation is carried out to prove the theoretical analysis. In Figure 5, the black dots are numerical solutions and the black line is the theoretical solution given by (21). The red shadow region is obtained by $Q<0$ (condition 1 ), which means that, in this region, the unstable solution cannot be obtained by simulation. While the red line decided by $P^{2}-4 Q=0$ (condition 2 ) illustrates that there are no periodical solutions between the two red lines, one can find that the theoretical analysis quite agrees with the numerical solutions.

When there are two stable steady state solutions coexisting, one of them has bigger amplitude than the other. Which kind of amplitude the system will act is decided by the initial conditions. In Figure 6, the $y$-axes mean the nondimensional displacement of the beam, the $\dot{y}$-axes mean the nondimensional velocity, then the domain of attraction is obtained by simulation, and the black region is the set of initial points leading to large amplitude, while the white region is the domain of attraction for small amplitude. 


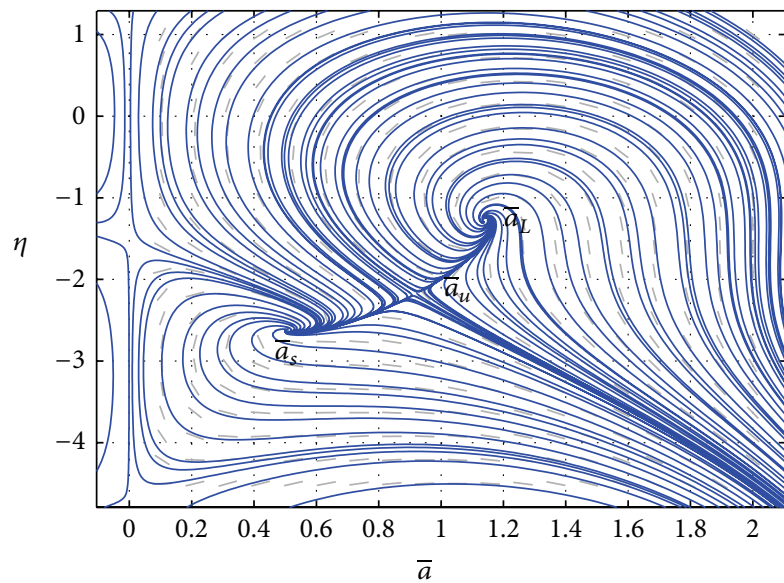

(a)

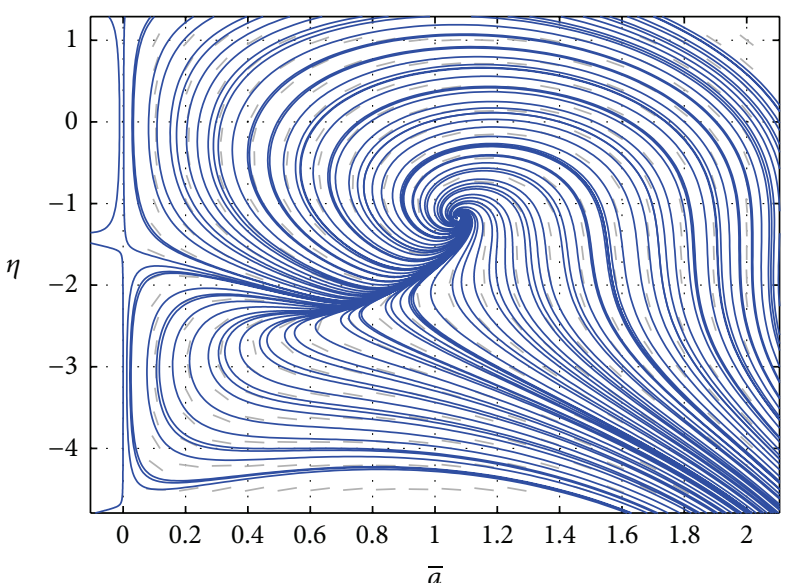

(b)

Figure 4: The steady state solutions of the noise-free equation (17) (a). $\sigma_{0}=0.22$ (b). $\sigma_{0}=0.18$.

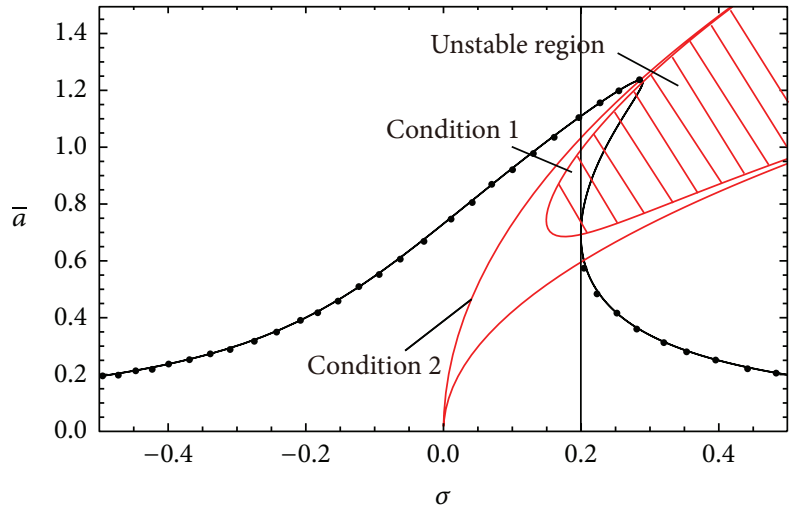

FIGURE 5: Frequency response (black dots: numerical simulations, black line: theoretical solution, red shadow: unstable region, and red lines: no periodical solution region).

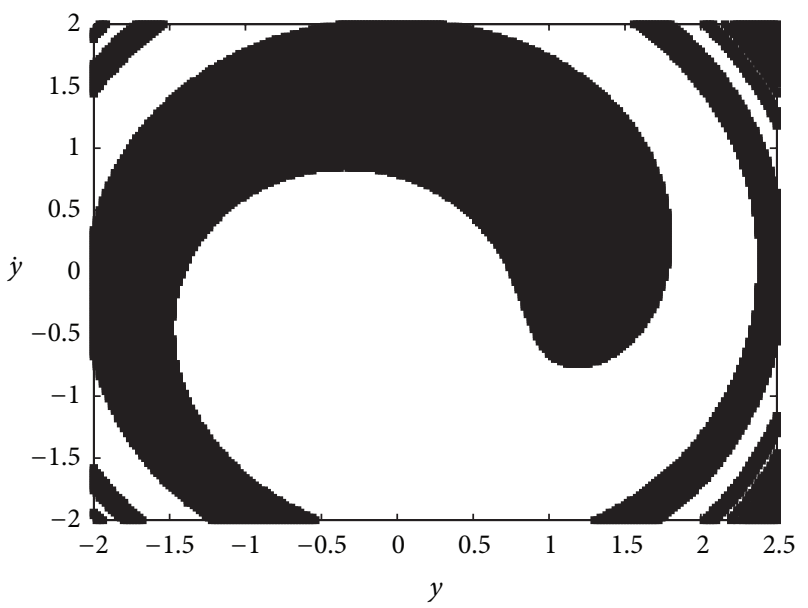

FIGURE 6: Domain of attraction to the large amplitude.
4.2. Noise Included Condition. For the noise included condition, that is, $\delta \neq 0$, the steady state response given by (18) is needed to study its stochastic response. The moment method [13-15] is an effective way to obtain the steady state moments $E[\Delta a], E\left[\Delta a^{2}\right]$. It is obvious that $\left(d E[\Delta a] / d T_{1}\right)=$ $\left(d E[\Delta \eta] / d T_{1}\right)=0$.

Tracking expectation on both sides of (20), one obtains

$$
E[\Delta a]=E[\Delta \eta]=0 .
$$

Similarly, we have $\left(d E\left[\Delta a^{2}\right] / d T_{1}\right)=\left(d E\left[\Delta \eta^{2}\right] / d T_{1}\right)=$ $\left(d E[\Delta a \Delta \eta] / d T_{1}\right)=0$. Applying Ito's rule of stochastic differentiation, one obtains

$$
\begin{gathered}
\left(\frac{3}{4} \beta \bar{a}^{2}-2 \mu\right) E\left[\Delta a^{2}\right]+\left(2 \bar{a} \sigma-\frac{3}{4} \alpha \bar{a}^{3}\right) E[\Delta a \Delta \eta]=0 \\
\left(\frac{9 \bar{a} \alpha}{4}-\frac{2 \sigma}{\bar{a}}\right) E[\Delta a \Delta \eta]+\left(\frac{\beta \bar{a}^{2}}{4}-2 \mu\right) E\left[\Delta \eta^{2}\right]=-\delta^{2} \\
\left(\frac{9 \alpha}{8} \bar{a}-\frac{\sigma}{\bar{a}}\right) E\left[\Delta a^{2}\right]+\left(\frac{\beta}{2} \bar{a}^{2}-2 \mu\right) E[\Delta a \Delta \eta] \\
+\left(-\frac{3 \alpha}{8} \bar{a}^{3}+\bar{a} \sigma\right) E\left[\Delta \eta^{2}\right]=0 .
\end{gathered}
$$

The solutions of (29) are

$$
\begin{aligned}
E\left[\Delta a^{2}\right]=\delta^{2}\left(3 \bar{a}^{3} \alpha-8 \bar{a} \sigma\right)^{2} & \\
\times\left(\left(4 \mu-\bar{a}^{2} \beta\right)[\right. & 3 \bar{a}^{4}\left(9 \alpha^{2}+\beta^{2}\right) \\
& -32 \bar{a}^{2}(\beta \mu+3 \alpha \sigma) \\
& \left.\left.+64\left(\mu^{2}+\sigma^{2}\right)\right]\right)^{-1},
\end{aligned}
$$




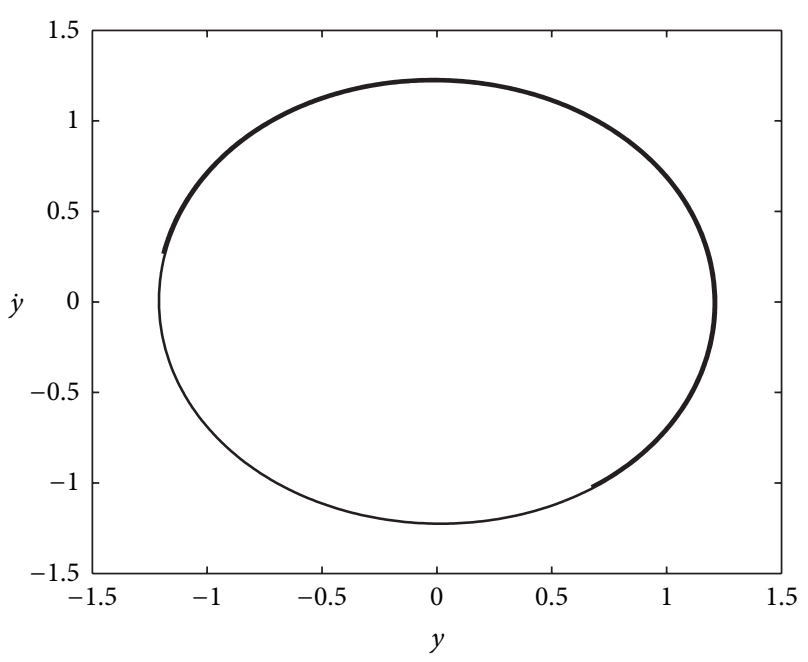

(a)

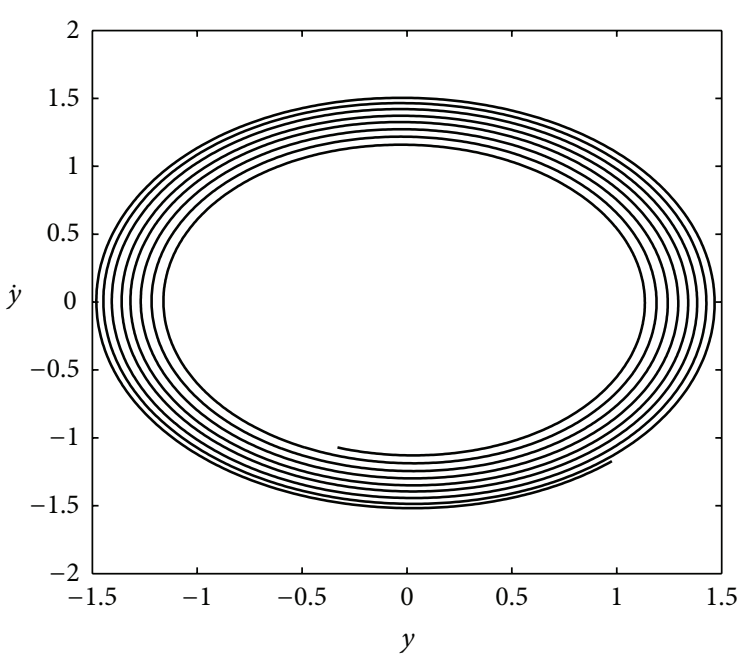

(b)

FIGURE 7: Phase plot for the noise-free and noise included system.

$$
\begin{gathered}
E[\Delta a \Delta \eta]=\left(\delta^{2}\left(3 \bar{a}^{2} \beta-8 \mu\right)\left(3 \bar{a}^{3} \alpha-8 \bar{a} \sigma\right)\right) \\
\times\left(( 4 \mu - \overline { a } ^ { 2 } \beta ) \left[3 \bar{a}^{4}\left(9 \alpha^{2}+\beta^{2}\right)\right.\right. \\
-32 \bar{a}^{2}(\beta \mu+3 \alpha \sigma) \\
\left.\left.+64\left(\mu^{2}+\sigma^{2}\right)\right]\right)^{-1}, \\
E\left[\Delta \eta^{2}\right]=\left(\delta ^ { 2 } \left[3 \bar{a}^{4}\left(9 \alpha^{2}+4 \beta^{2}\right)\right.\right. \\
\quad \times\left(( 4 \mu - \overline { a } ^ { 2 } \beta ) \left[3 \bar{a}^{4}\left(9 \alpha^{2}+\beta^{2}\right)\right.\right. \\
-32 \bar{a}^{2}(\beta \mu+3 \alpha \sigma) \\
\left.\left.+64\left(\mu^{2}+\sigma^{2}\right)\right]\right)^{-1} .
\end{gathered}
$$

Combining (28) and (30), one obtains

$$
\begin{array}{ll}
E[a]=\bar{a}, & E\left[a^{2}\right]=\bar{a}^{2}+E\left[\Delta a^{2}\right] \\
E[\eta]=\bar{\eta}, & E\left[\eta^{2}\right]=\bar{\eta}^{2}+E\left[\Delta \eta^{2}\right] .
\end{array}
$$

Form (30) it is easy to see that, for $E\left[\Delta a^{2}\right] \geq 0, E\left[\Delta \eta^{2}\right] \geq 0$, there must be $4 \mu-\bar{a}^{2} \beta \geq 0$ and $3 \bar{a}^{2}\left(9 \alpha^{2}+\beta^{2}\right)-32(\beta \mu+$ $\left.3 \alpha \sigma_{0}\right)+64\left(\mu^{2}+\sigma_{0}{ }^{2}\right)$. One finds that the existing condition for second-order moment is the same as the stability condition for the first-order moment. By the method proposed by Zhu [16], the numerical simulation is carried out with small noise density $\bar{\delta}=0.05$. When $\sigma_{0}=0.22$, the results are shown in Figures 7 and 8 . For comparison, the noise-free condition is also given in Figure 7 . The initial points chosen as $(1,2, .5)$ can lead to the phase portraits as Figures 7(a) and 7(b). For the noise-free condition, the phase portrait is a limit cycle (see Figure $7(\mathrm{a})$ ), while, for the noise included system, the limit cycle is a diffused one (see Figure 7(b)).

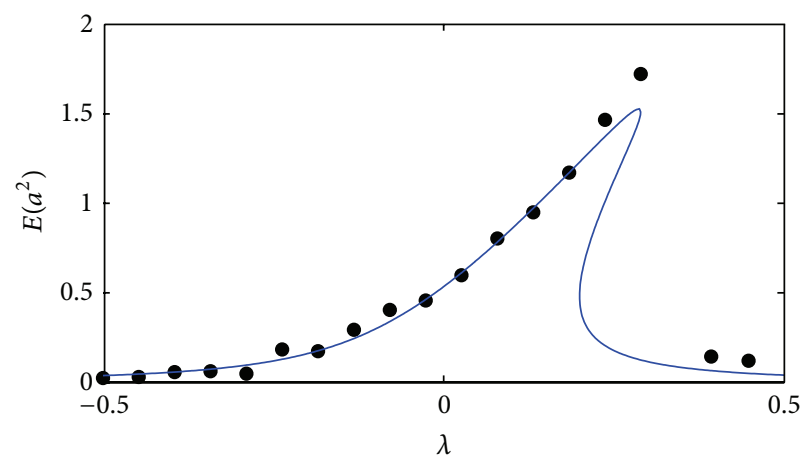

FIGURE 8: The frequency response with noise (black dots: numerical simulations and line: theoretical results).

Figure 8 shows the frequency response with noise of this system. Obviously, with small enough $\bar{\delta}$, the theoretical analysis also approximately agrees with the numerical test. It is found when the exciting frequency is low, the numerical solution agrees with the theoretical solution and when the exciting frequency becomes larger, the numerical solution seems to be not so good compared with the theoretical solution.

\section{Summary and Conclusion}

In this paper, a Van-der-Pol hysteretic cycle is used to simulate the hysteretic character of SMA, which is suitable and convenient for modeling. Then, a nonlinear vibration model of simply supported SMA beam under narrow band noise excitation is built and approximately solved by the multiple scale method. The steady state responses are obtained for both noise-free condition and noise included condition, respectively. After that, the stability of the steady state is analyzed. Some complex dynamical phenomena can be observed, which is helpful for industrial application. 
When the strength of the disturbing noise is small enough, the original system can be simplified to an Itotype differential equation. The singularity theory is applied to study how the parameters influence the number of stationary solutions. It is found that, with some proper parameters there exist three steady state solutions; two of them are stable and realizable, while the other one is unstable. The initial conditions decide which kind of amplitude can appear. The moment method is used to obtain the first-order and secondorder moments of the random steady state responses. Similar results are found compared with the deterministic condition. But the difference is that the limit cycle is diffused by the noise. The results of numerical simulation approximately agree with the analysis.

\section{Conflict of Interests}

The author declares that there is no conflict of interest regarding the publication of this paper.

\section{Acknowledgments}

This work was supported by the National Natural Science Foundation of China (Grant no. 11272229 and Grant no. 11302144) and the Science Foundation of Tianjin education committee (Grant no. 20120902).

\section{References}

[1] J. G. Boyd and D. C. Lagoudas, "A thermodynamical constitutive model for shape memory materials-part I: the monolithic shape memory alloy," International Journal of Plasticity, vol. 12, no. 6, pp. 805-873, 1996.

[2] E. J. Graesser and F. A. Cozzarelli, "Proposed three-dimensional constitutive model for shape memory alloys," Journal of Intelligent Material Systems and Structures, vol. 5, no. 1, pp. 78-89, 1994.

[3] F. Auricchio and J. Lubliner, "A uniaxial model for shapememory alloys," International Journal of Solids and Structures, vol. 34, no. 27, pp. 3601-3618, 1997.

[4] Z. Q. Wu and Z. H. Zhang, "Force-displacement characteristics of simply supported beam laminated with shape memory alloys," Acta Mechanica Sinica, vol. 27, no. 6, pp. 1065-1070, 2011.

[5] M. Collet, E. Foltete, and C. Iexcellent, "Analysis of the behavior of a shape memory alloy beam under dynamical loading," European Journal Mechanics, vol. 20, pp. 615-630, 2001.

[6] S. M. T. Hashemi and S. E. Khadem, "Modeling and analysis of the vibration behavior of a shape memory alloy beam," International Journal of Mechanical Sciences, vol. 48, no. 1, pp. 44-52, 2006.

[7] M. A. Savi, P. M. Pacheeo, and M. B. Braga, "Chaos in shape memory two-bar truss," International Journal of Non-Linear Mechanics, vol. 37, no. 1, pp. 1387-1395, 2002.

[8] Q. Q. Zhang, Y. H. Li, and J. Yao, "Dynamic stability and chaotic motion of a shape memory alloy beam," Journal of Sichuan University, vol. 36, no. 5, pp. 30-34, 2004 (Chinese).

[9] Z. W. Zhu, W. Y. Xie, and J. Xu, "Nonlinear dynamic characteristics of SMA simply supported beam in axial stochastic excitation," Journal of Vibro Engineering, vol. 14, no. 3, pp. 10591063, 2012.
[10] X. Yan and J. Nie, "Response of SMA superelastic systems under random excitation," Journal of Sound and Vibration, vol. 238, no. 5, pp. 893-901, 2000.

[11] B. Wu, K. X. Sun, H. Li, and W.-S. Tang, "Experimental research on mechanical properties of shape memory alloy," Journal of Earthquake Engineering and Engineering Vibration, vol. 19, no. 2, pp. 100-111, 1999 (Chinese).

[12] M. Golubitsky and D. G. Scheeffer, "Singularities and groups in bifurcation theory," Applied Mathematical Science, pp. 186-190, 1985.

[13] H. W. Rong, X. D. Wang, G. Meng, W. Xu, and T. Fang, "Response of nonlinear oscillator under narrow-band random excitation," Applied Mathematics and Mechanics, vol. 24, no. 7, pp. 817-825, 2003.

[14] W. Q. Zhu, M. Q. Lu, and Q. T. Wu, "Stochastic jump and bifurcation of a duffing oscillator under narrow-band excitation," Journal of Sound and Vibration, vol. 165, no. 2, pp. 285-304, 1993.

[15] H. W. Rong, W. Xu, and T. Fang, "Principal response of duffing oscillator to combined deterministic and narrow-band random parametric excitation," Journal of Sound and Vibration, vol. 210, no. 4, pp. 483-515, 1998.

[16] W. Q. Zhu, Random Vibration, Science Press, Beijing, China, 1992 (Chinese). 


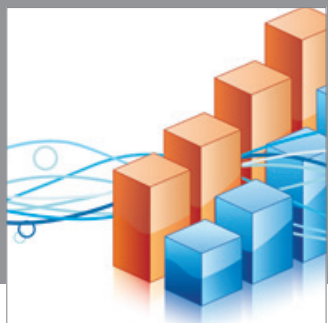

Advances in

Operations Research

mansans

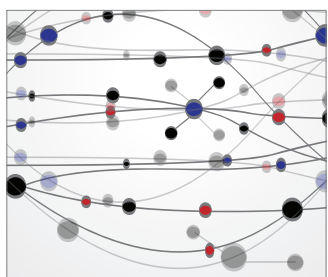

The Scientific World Journal
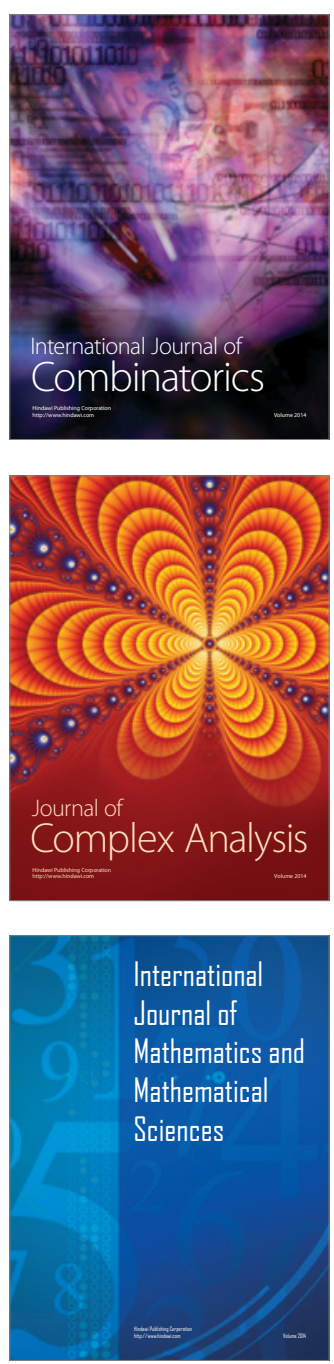
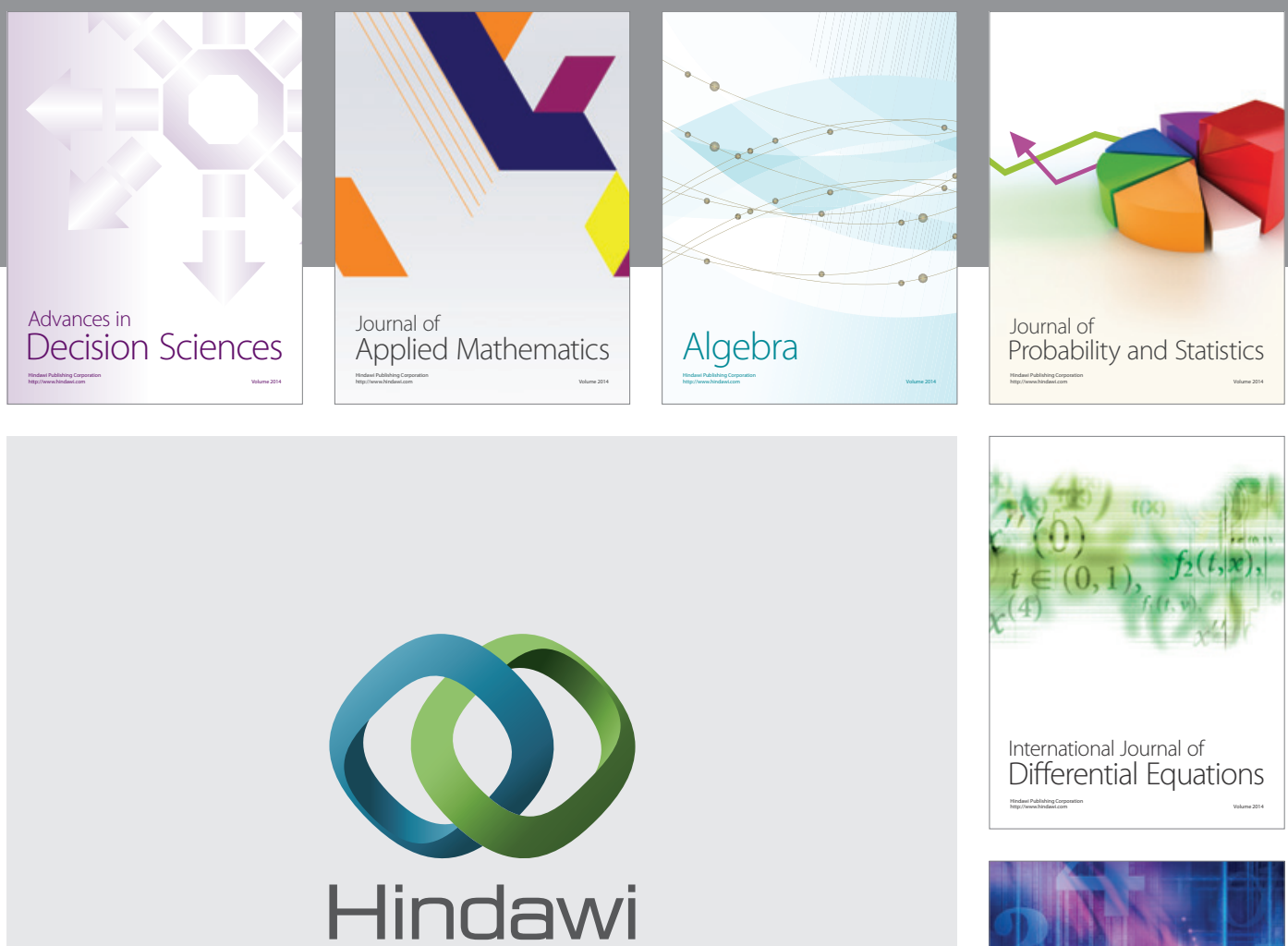

Submit your manuscripts at http://www.hindawi.com
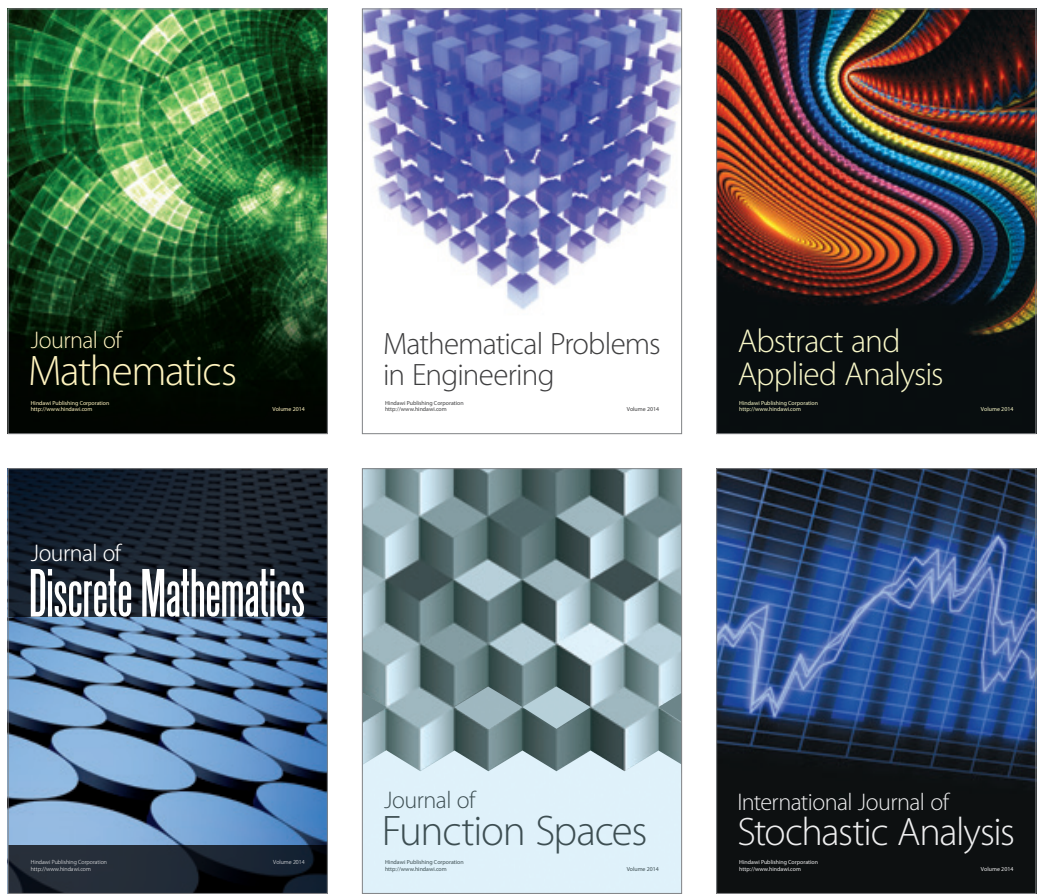

Journal of

Function Spaces

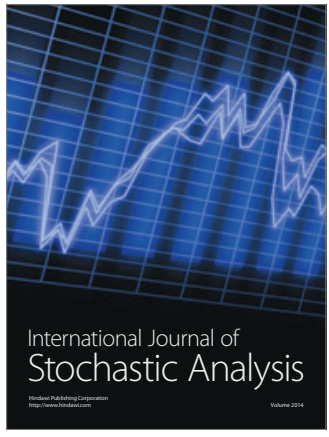

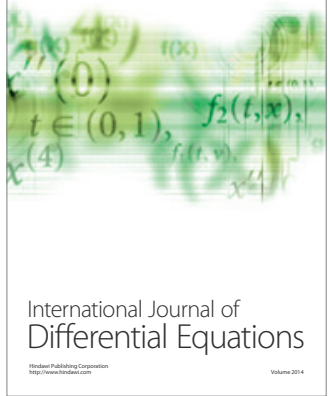
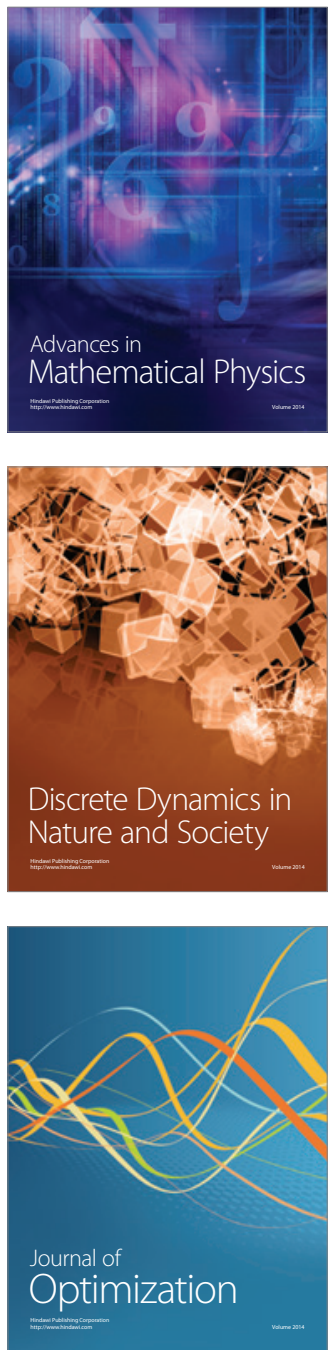